PROCEEDINGS OF THE

AMERICAN MATHEMATICAL SOCIETY

Volume 132, Number 8, Pages 2273-2280

S 0002-9939(04)07471-4

Article electronically published on March 25, 2004

\title{
A LIMIT-POINT CRITERION FOR A CLASS OF STURM-LIOUVILLE OPERATORS DEFINED IN $L^{p}$ SPACES
}

\author{
R. C. BROWN \\ (Communicated by Carmen C. Chicone)
}

\begin{abstract}
Using a recent result of Chernyavskaya and Shuster we show that the maximal operator determined by $M[y]=-y^{\prime \prime}+q y$ on $[a, \infty), a>-\infty$, where $q \geq 0$ and the mean value of $q$ computed over all subintervals of $\mathbb{R}$ of a fixed length is bounded away from zero, shares several standard "limit-point at $\infty$ " properties of the $L^{2}$ case. We also show that there is a unique solution of $M[y]=0$ that is in all $L^{p}[a, \infty), p=[1, \infty]$.
\end{abstract}

\section{INTRODUCTION}

In 3 Chernyavskaya and Shuster have determined necessary and sufficient conditions for the symmetric differential expression $M[y]=-y^{\prime \prime}+q y$ where $q \geq 0$ and $q$ is locally Lebesgue integrable to be "correctly solvable" in $L^{p}(\mathbb{R})$. This concept means:

(i) for every $f \in L^{p}(\mathbb{R}), p \in[1, \infty]$, there is a unique solution $y_{p} \in L^{p}(\mathbb{R})$ of $M[y]=f$

(ii) $y_{p}$ satisfies the inequality

$$
\left\|y_{p}\right\|_{p, \mathbb{R}} \leq c(p)\left\|M\left[y_{p}\right]\right\|_{p, \mathbb{R}} .
$$

A main result of [3] is

Theorem A. $M$ is correctly solvable in $L^{p}(\mathbb{R}), p \in[1, \infty]$, if and only if there exists $a \in(0, \infty)$ such that

$$
q_{0}(a):=\inf _{x \in \mathbb{R}} \int_{x-a}^{x+a} q d t>0 .
$$

Two corollaries to Theorem A are:

(i) $M$ is correctly solvable in $L^{p}(\mathbb{R}), p \in[1, \infty]$, if $q \geq k>0$ on $\mathbb{R}$;

(ii) $M$ is not correctly solvable in $L^{p}(\mathbb{R})$ for any $p \in[1, \infty)$ if $q \in L(-\infty, 0)$ or $q \in L(0, \infty)$.

Received by the editors December 18, 2002.

2000 Mathematics Subject Classification. Primary 47E05, 34C11, 34B24; Secondary 34C10.

Key words and phrases. Second-order differential operators of symmetric form in $L^{p}$ spaces, correct solvability, limit-point, $L^{p}$ solutions.

(C)2004 American Mathematical Society 
We assume throughout that $M$ satisfies (1.2) and shall then show that the correct solvability of $M$ implies two interesting conclusions holding for $p \in[1, \infty]$, one of which generalizes the fact that the restriction of $M$ to the half-line $I=[a, \infty)$ is limit-point at $\infty$ in $L^{2}\left(I_{a}\right)$.

Before stating them however, we need some preliminaries. If $X$ is a Banach space with norm $\|(\cdot)\|$, e.g. $L^{p}(\mathbb{R}), p \in[1, \infty]$, and $T: X \rightarrow X$ is an operator with dense domain $\mathcal{D} \subset X$ we write $R(T)$ and $N(T)$ for the range and null space of $T$. If $X^{*}$ is the dual of $X$, e.g. $L^{p^{\prime}}\left(I_{a}\right), p^{\prime}=p / p-1$, and $\left[x, x^{*}\right]$ signifies $\overline{x^{*}}(x)$ for $x \in X$ and $x^{*} \in X^{*}$, we consider the set of pairs $G\left(T^{*}\right):=\left(z, z^{\prime}\right) \in X^{*} \times X^{*}$ such that

$$
[T(y), z]=\left[y, z^{\prime}\right] .
$$

The density of $\mathcal{D}$ implies that $G\left(T^{*}\right)$ determines an operator $T^{*}$ called the adjoint of $T$ such that $T^{*}(z)=z^{\prime}$. If $T: X^{*} \rightarrow X^{*}$ has a domain $\mathcal{D}^{*}$ that is total over $X$ (i.e., $\left[x, x^{*}\right]=0$ for all $x \in X$ and $x^{*} \in \mathcal{D}^{*} \Longrightarrow x=0$ ), then the set of pairs $\left(z, z^{\prime}\right) \in X \times X$ satisfying (1.3) also determines an operator, which we also denote by $T^{*}$, and call the adjoint of $T$ in $X$. In either case $T^{*}$ is closed and

$$
[T(y), z]=\left[y, T^{*}(z)\right]
$$

for all $y \in \mathcal{D}, z \in \mathcal{D}^{*}$. Furthermore, if $M$ is a subspace of $X$ and $M^{*}$ is a subspace of $X^{*}$, then

$$
\begin{aligned}
M^{\perp} & :=\left\{x^{*} \in X^{*}:\left[x, x^{*}\right]=0, \quad \forall x \in M\right\}, \\
{ }^{\perp} M^{*} & :=\left\{x \in X:\left[x, x^{*}\right]=0, \quad \forall x^{*} \in M^{*}\right\},
\end{aligned}
$$

and if $X$ is reflexive, i.e., the natural mapping of $X$ to $\left(X^{*}\right)^{*} \equiv X^{* *}$ is an isomorphism, then $\left(M^{*}\right)^{\perp}={ }^{\perp} M$. If we set $G(T):=\{(y, T(y)): y \in \mathcal{D}\}$ and $G(-T):=\{(y,-T(y)): y \in \mathcal{D}\}$, then $G\left(T^{*}\right)=G(-T)^{\perp} \subset X^{*} \times X^{*}$.

It follows from these definitions and (1.3) (see, e.g., Kato, [7, Problem 5.27, p. 168], Rudin, [11] Theorem 4.7] and Goldberg, [5, Theorem IV.1.3] that when $T$ is closed,

$$
\begin{aligned}
& R(T)^{\perp}=N\left(T^{*}\right), \quad{ }^{\perp} R\left(T^{*}\right)=N(T), \\
& \overline{R(T)}={ }^{\perp} N\left(T^{*}\right), \quad \overline{R\left(T^{*}\right)}=N(T)^{\perp} .
\end{aligned}
$$

We are interested in the operators determined by $M$ but on the half-line $I_{a}=$ $[a, \infty),-\infty<a$, rather than on $\mathbb{R}$. This parallels a common situation in the Hilbert space theory of $M$ and allows study of the $L^{p}$ solutions of $M[y]=0$ on $I_{a}$, which obviously cannot exist on $\mathbb{R}$ if $M$ is correctly solvable.

If $A C_{\mathrm{loc}}\left(I_{a}\right)$ denotes the functions that are locally absolutely continuous on $I_{a}$, we set

$$
\{y, z\}(t):=y^{\prime}(t) \bar{z}(t)-y(t) \bar{z}^{\prime}(t)
$$

for $y, z \in A C_{\mathrm{loc}}\left(I_{a}\right)$ and define the following operators and domains in $L^{p}\left(I_{a}\right)$. 
Definition. For $p \in[1, \infty]$ assume that $q \in L_{\mathrm{loc}}^{\infty}\left(I_{a}\right) \subset L_{\mathrm{loc}}\left(I_{a}\right){ }^{1}$ and let $T_{0, p}^{\prime}, T_{p}$, and $T_{0, p}$ be the operators determined by $M$ on

$$
\begin{aligned}
\mathcal{D}_{0, p}^{\prime} & :=\left\{y \in C_{0}^{\infty}\left(I_{a}\right)\right\}, \\
\mathcal{D}_{p} & :=\left\{y \in L^{p}\left(I_{a}\right): y^{\prime} \in A C_{\mathrm{loc}}\left(I_{a}\right) ; M[y] \in L^{p}\left(I_{a}\right)\right\}, \\
\mathcal{D}_{0, p} & :=\left\{y \in \mathcal{D}_{p}: y(a)=y^{\prime}(a)=0 ; \lim _{t \rightarrow \infty}\{y, z\}(t)=0, \forall z \in \mathcal{D}_{p}\right\} .
\end{aligned}
$$

We call $T_{0, p}^{\prime}, T_{0, p}$ respectively the "preminimal" and "minimal" operators, and $T_{p}$ the "maximal" operator determined by $M$. These operators have the following properties.

Theorem B. For the cases $p \in(1, \infty), p=1$, or $p=\infty$ set $p^{\prime}:=p /(p-1)$, $p^{\prime}=\infty$, or $p^{\prime}=1$. Then

(i) $T_{0, p}$ and $T_{p}$ are closed operators;

(ii) $\left[T_{p}(y), z\right]=\lim _{t \rightarrow \infty}\{y, z\}(t)-\{y, z\}(a)+\left[y, T_{p^{\prime}}\right]$;

(iii) $T_{p}^{*}=T_{0, p^{\prime}}$ and $T_{0, p}^{*}=T_{p^{\prime}}$;

(iv) $R\left(T_{0, p}\right)^{\perp}=N\left(T_{p^{\prime}}\right)$ and ${ }^{\perp} N\left(T_{p^{\prime}}\right)=\overline{R\left(T_{0, p}\right)}$;

(v) $N\left(T_{0, p}\right)=\{0\}$.

Moreover, for $p \in(1, \infty], T_{0, p}^{\prime}$ is closable and $\overline{T_{0, p}^{\prime}}=T_{0, p}$.

Proofs of (i)-(v), the last statement, as well as more general results may be found in one of [5, Chapter VI], [10, or [2]. (iv) is a consequence of the fact that solutions of $M[y]=0$ under prescribed initial conditions are unique. The $L^{2}$ theory is throughly treated in 8 .

We are now in a position to state our two principal results.

Theorem 1. If $q \in L_{l o c}^{\infty}$ and satisfies (1.2) and $p \in[1, \infty]$, then $M$ is " $p$ limitpoint" ( $p L P)$ at $\infty$ in the sense that

(i) $\operatorname{dim}\left(\frac{\mathcal{D}_{p}}{\mathcal{D}_{0, p}}\right)=2$ and $\operatorname{dim}\left(\frac{R\left(T_{p}\right)}{R\left(T_{0, p}\right.}\right)=1$;

(ii) $\operatorname{dim} N\left(T_{p}\right)=1$;

(iii) for all $y \in \mathcal{D}_{p}$ and $z \in \mathcal{D}_{p^{\prime}}$ we have $\lim _{t \rightarrow \infty}\{y, z\}(t)=0$.

Theorem 2. If $y_{1}$ denotes the principal or "small" solution of $M[y]=0$, then $y_{1} \in L^{p}\left(I_{a}\right)$ for all $p \in[1, \infty]$.

Remarks. (i) Theorem 1 is an extension to all $p \in[1, \infty]$ and to a more general $q$ of the fact that $M$ is $L P$ at $\infty$ when $q \geq k>0$ and $p=2$.

(ii) In the case when $q \geq k>0$ a direct argument can be given to show that $M[y]=0$ has exponentially growing and exponentially decaying solutions. Read 9] has extended this by showing that the same is true if

$$
\liminf _{x \rightarrow \infty} \int_{x}^{x+a} q^{1 / 2} d t>a L
$$

for positive constants $a$ and $L$. Clearly (1.6) implies (1.2) of Theorem A for the extension $\hat{q}_{1}$ of $q$ obtained by setting $\hat{q}_{1}(t)=1$ for $t<a$. In this case or if $q$

\footnotetext{
${ }^{1}$ By extending the procedure of Naimark [8, $\left.\$ 17\right]$, used to define maximal and minimal operators in the $L^{2}$ case, it is almost certain that $q$ need only be locally integrable. However, in this case, $\mathcal{D}_{0, p}^{\prime}$ need not contain any member of $C_{0}^{\infty}\left(I_{a}\right)$ and has to be redefined as the subspace of $\mathcal{D}$ having compact support on $I_{a}$. The density of $\mathcal{D}_{0, p}^{\prime}$ must then be shown by an independent argument. Because of its technical complications we will not pursue this approach here.
} 
is bounded away from zero, Theorem 2 is trivially true since $y_{1}$ is exponentially decreasing. Under the condition (1.2), however, Theorem 2 seems to be new.

(iii) In the $L^{2}$ theory $M$ is said to be strong limit-point $(S L P)$ or Dirichlet $(D)$ at $\infty$ if $\lim _{t \rightarrow \infty} y^{\prime} \bar{z}=0$ for all $y, z \in \mathcal{D}_{2}$, or if $y^{\prime}$ and $q^{1 / 2} y \in L^{2}\left(I_{a}\right)$ for all $y \in \mathcal{D}_{2}$. It is known [1] that $D \Rightarrow S L P$ and that both $S L P$ and $D$ hold if $q \geq k>0$. It would be interesting to see if either $S L P$ or $D$ might be profitably extended to the $L^{p}$ setting.

\section{Proofs of Theorems 1 and 2}

Lemma 1. For $p \in[1, \infty], R\left(T_{p}\right)=L^{p}\left(I_{a}\right)$ and $T_{0, p}$ has closed range.

Proof. Since $q$ is defined only on $I_{a}$, to apply Theorem A we consider the extension $\hat{q}_{1}$. Similarly if $f \in L^{p}\left(I_{a}\right)$, we construct an extension $\hat{f}$ to $\mathbb{R}$ by setting $\hat{f}(t)=0$ for $t<a$. By Theorem A there is a unique $\hat{y} \in L^{p}(\mathbb{R})$ such that $M[y]=\hat{f}$. The restriction $y$ of $\hat{y}$ to $I_{a}$ is evidently in $\mathcal{D}_{p}$ and satisfies $M[y]=f$, showing that $T_{p}$ is onto $L^{p}\left(I_{a}\right)$. If $R\left(T_{0}, p\right)$ is not closed, by the Closed Range Theorem the inverse of $T_{0, p}$ is unbounded. Therefore there are sequences $\left\{y_{n}\right\} \subset \mathcal{D}_{0, p},\left\{f_{n}\right\} \subset R\left(T_{0, p}\right)$ such that $f_{n} \rightarrow 0,\left\|y_{n}\right\|=1$, and $M\left[y_{n}\right]=f_{n}$. Let $\hat{y}_{n}$ and $\hat{f}_{n}$ be the extensions of $y_{n}$ and $f_{n}$ to $\mathbb{R}$ defined as above. Applying Theorem A again there is a unique $\hat{z}_{n}$ for each $\hat{f}_{n}$ such that $M\left[\hat{z}_{n}\right]=f_{n}$ and

$$
\begin{aligned}
\left\|\hat{z}_{n}\right\|_{p, \mathbb{R}} & \leq c_{p}\left\|\hat{f}_{n}\right\|_{p, \mathbb{R}} \\
& \leq c_{p}\left\|f_{n}\right\|_{p, \mathbb{R}} .
\end{aligned}
$$

Therefore if $z_{n}$ is the restriction of $\hat{z}_{n}$ to $I_{a}$ we have also that $\left\|z_{n}\right\|_{p, I_{a}} \leq c_{p}\left\|f_{n}\right\|_{p, I_{a}}$. Hence since $f_{n} \rightarrow 0$, so does $z_{n}$. Because $M\left[z_{n}\right]=f_{n}=M\left[y_{n}\right]$ we have that $z_{n}-y_{n} \in N\left(T_{p}\right)$. Moreover, $1 / 2 \leq\left\|z_{n}-y_{n}\right\|_{p, I_{a}} \leq 3 / 2$ for sufficiently large $n$ because $\left\|y_{n}\right\|_{p, I_{a}}=1$ and $z_{n} \rightarrow 0$. If $N\left(T_{p}\right)=\{0\}$, then $z_{n}=y_{n}$, which is impossible. If $N\left(T_{p}\right)=\operatorname{span}\{u\}$ where $\|u\|=1$, then $z_{n}-y_{n}=k_{n} u$. Since the $k_{n}$ lie in the bounded interval $[1 / 2,3 / 2]$ for sufficiently large $n$, there must be a subsequence $k_{n_{i}} \rightarrow k \neq 0$. It follows that $y_{n_{i}} \rightarrow-k u$. Because $T_{0, p}$ is closed, $u \in \mathcal{D}_{0, p}$. But since the null space of $T_{0, p}$ is trivial, $u=0$, contradicting our assumption. The case $N\left(T_{p}\right)=\operatorname{span}\left\{u_{1}, u_{2}\right\}$ is handled by a similar argument.

The following three results are standard, but since some are difficult to find in the literature in the form stated we include proofs.

Lemma 2. If $X$ is a Banach space, $X^{*}$ is its dual, and $M$ is a finite-dimensional subspace of $X$, then

$$
\operatorname{dim}\left(\frac{X^{*}}{M^{\perp}}\right)=\operatorname{dim} M
$$

Proof. Let $\left\{m_{i}\right\}, i=1, \ldots, n$, be a basis for $M$. By the Hahn-Banach theorem we can find $f_{i} \in X^{*}$ such that $f_{i}\left(m_{j}\right)=\delta_{i j}, 1 \leq i, j \leq n$. We claim that $\left\{f_{i}\right\}$, $i=1, \ldots, n$, is a linearly independent set $\bmod M^{\perp}$. For if $c_{1} f_{1}+\cdots+c_{n} f_{n} \in M^{\perp}$ where not all the $c_{i}$ are zero we have that for any particular (and therefore all) $c_{j}$ that

$$
c_{j}=c_{j} f_{j}\left(m_{j}\right)=\left(\sum_{i=1}^{n} c_{i} f_{i}\right)\left(m_{j}\right)=0,
$$


which is a contradiction. It follows that

$$
\operatorname{dim} M \leq \operatorname{dim}\left(\frac{X^{*}}{M^{\perp}}\right) .
$$

If the inequality is strict we can find $f \in X^{*}$ such that $S=\left\{f_{1}, \ldots, f_{n}, f\right\}$ is linearly independent $\bmod M^{\perp}$. Consider

$$
\psi:=f-\sum_{i=1}^{m} f\left(m_{i}\right) f_{i} .
$$

Since $f \notin M^{\perp}$, not all the $f\left(m_{i}\right)=0$. However, $\psi\left(m_{i}\right)=0$ for $i=1, \ldots, n$, showing that $\psi \in M^{\perp}$ so that $S$ is linearly dependent $\bmod M^{\perp}$ and thus contradicting our assumption.

Lemma 3. Suppose that $X$ and its dual $X^{*}$ are Banach spaces, and let $T_{2} \subset T_{1}$ be densely defined operators $X \rightarrow X$ with domains $\mathcal{D}_{1}$ and $\mathcal{D}_{2}$, and let $T_{1}^{*} \subset T_{2}^{*}$ be their adjoints with domains $\mathcal{D}_{1}^{*}$ and $\mathcal{D}_{2}^{*}$. If $\operatorname{dim}\left(\mathcal{D}_{1} / \mathcal{D}_{2}\right)=n<\infty$, then

$$
\operatorname{dim}\left(\frac{\mathcal{D}_{1}}{\mathcal{D}_{2}}\right)=\operatorname{dim}\left(\frac{\mathcal{D}_{2}^{*}}{\mathcal{D}_{1}^{*}}\right) .
$$

Proof. The technique is similar to that of Lemma 2 First note that

$$
\operatorname{dim}\left(\frac{\mathcal{D}_{1}}{\mathcal{D}_{2}}\right)=\operatorname{dim}\left(\frac{G\left(-T_{1}\right)}{G\left(-T_{2}\right)}\right) \quad \text { and } \quad \operatorname{dim}\left(\frac{\mathcal{D}_{2}^{*}}{\mathcal{D}_{1}^{*}}\right)=\operatorname{dim}\left(\frac{G\left(T_{2}^{*}\right)}{G\left(T_{1}^{*}\right)}\right) .
$$

Let $\nu_{i}=\left(\alpha_{i},-T_{1}\left(\alpha_{i}\right)\right), i=1, \ldots, n$, be a linearly independent set $\bmod G\left(-T_{2}\right)$. By the Hahn-Banach Theorem we can find

$$
\psi_{j}=\left(u_{j}, v_{j}\right) \in\left(G\left(-T_{2}\right)\right)^{\perp} \equiv G\left(T_{2}^{*}\right) \subset X^{*} \times X^{*}, \quad j=1, \ldots, n,
$$

such that $\psi_{j}\left(\nu_{i}\right) \equiv u_{j}\left(\alpha_{i}\right)+v_{j}\left(-T_{1}\left(\alpha_{i}\right)\right)=\delta_{i j}$. Now $\left\{\psi_{j}\right\}, j=1, \ldots, n$, is a linearly independent set $\bmod G\left(T_{1}^{*}\right)$, for if

$$
\eta=\sum_{j=1}^{n} c_{j} \psi_{j} \in G\left(T_{1}^{*}\right),
$$

then $\eta\left(\nu_{i}\right)=0=c_{i}$ for $i=1, \ldots, n$. This shows that $\operatorname{dim}\left(\mathcal{D}_{1} / \mathcal{D}_{2}\right) \leq \operatorname{dim}\left(\mathcal{D}_{2}^{*} / \mathcal{D}_{1}^{*}\right)$. But if $\left\{\psi_{1}, \ldots, \psi_{n}, \psi\right\}, \psi \in G\left(T_{2}^{*}\right)$ is linearly independent $\bmod G\left(T_{1}^{*}\right)$, this contradicts the fact that $\psi-\sum_{i=1}^{n} d_{i} \psi_{i} \in G\left(T_{1}^{*}\right)$ where $d_{i}=\psi\left(\nu_{i}\right)$.

Lemma 4. Suppose that $X$ is a Banach space and $X^{*}$ is its dual, and let $T_{1}$ : $X^{*} \rightarrow X^{*}$ be an operator with domain $\mathcal{D}_{1}$ and $T_{2}$ be a one-to-one restriction of $T_{1}$ with closed range having domain $\mathcal{D}_{2} \subset \mathcal{D}_{1}$. If $\operatorname{dim} N\left(T_{1}\right)<\infty$ and $\operatorname{dim}{ }^{\perp} R\left(T_{2}\right) \subseteq$ $X<\infty$, then

$$
\operatorname{dim}\left(\frac{\mathcal{D}_{1}}{\mathcal{D}_{2}}\right)=\operatorname{dim} N\left(T_{1}\right)+\operatorname{dim}^{\perp} R\left(T_{2}\right) .
$$

Proof. In Lemma 2 we identify $M$ with ${ }^{\perp} R\left(T_{2}\right)$. Since $R\left(T_{2}\right)$ is closed, $M^{\perp}=$ $R\left(T_{2}\right)$. We can conclude that

$$
\operatorname{dim}\left(\frac{R\left(T_{1}\right)}{R\left(T_{2}\right)}\right)=\operatorname{dim}^{\perp} R\left(T_{2}\right) .
$$

If $k=\operatorname{dim}{ }^{\perp} R\left(T_{2}\right)$, we can find nonzero elements $f_{1}, \ldots, f_{k}$ in $R\left(T_{1}\right)$ that are linearly independent $\bmod R\left(T_{2}\right)$. Suppose $y_{i} \in \mathcal{D}_{1}$ and $M\left[y_{i}\right]=f_{i}, i=1, \ldots, k$. 
Then it is easily verified that $S_{1}=\left\{y_{1}, \ldots, y_{k}\right\}$ is a linearly independent set mod $\mathcal{D}_{2}$ (for otherwise a linear combination of the $f_{i}$ would be in $R\left(T_{2}\right)$ ). Let $S_{2}=$ $\left\{z_{1}, \ldots, z_{m}\right\}$ be a basis of $N\left(T_{1}\right)$. We claim that $S_{1} \cup S_{2}$ is linearly independent $\bmod \mathcal{D}_{2}$. For assume that $\eta=\eta_{1}+\eta_{2} \in \mathcal{D}_{2}$ where

$$
\eta_{1}=\sum_{i=1}^{k} c_{i} y_{i}, \quad \eta_{2}=\sum_{i=1}^{m} d_{i} z_{i}
$$

and not all the coefficients vanish. In particular, under this assumption, since $N\left(T_{1}\right) \cap \mathcal{D}_{2}=\{0\}$, not all the $c_{i}=0$ vanish, for otherwise $\eta_{2} \neq 0$ and $\eta_{2} \in$ $\mathcal{D}_{2} \cap N\left(T_{1}\right)$. But then

$$
T_{1}(\eta)=T_{1}\left(\eta_{1}\right)=\sum_{i=1}^{k} c_{i} f_{i} \in R\left(T_{2}\right),
$$

contradicting the linear independence of the $f_{i} \bmod R\left(T_{2}\right)$. It follows that

$$
\operatorname{dim}\left(\frac{\mathcal{D}_{1}}{\mathcal{D}_{2}}\right) \geq \operatorname{dim} N\left(T_{1}\right)+\operatorname{dim}^{\perp} R\left(T_{2}\right)=m+k .
$$

Suppose that we can adjoin an element $u \in \mathcal{D}_{1}$ to $S_{1} \cup S_{2}$ so that $S_{3}=\left(S_{1} \cup S_{2}\right) \cup\{u\}$ is linearly independent $\bmod \mathcal{D}_{2}$. Since $N\left(T_{1}\right)$ is finite dimensional, it is complemented in $X$, and so $u$ can be written uniquely as a sum $u_{1}+u_{2}$ where $u_{1} \in N\left(T_{1}\right)$ and $u_{2} \in\left(\mathcal{D}_{1} \backslash N\left(T_{1}\right)\right) \cup\{0\}$. If $\eta_{3}$ is an arbitrary linear combination of elements of $S_{3}$ with a nonzero coefficient $d$ of $u$, then

$$
T_{1}\left(\eta_{3}\right)=\sum_{i=1}^{k} c_{i} f_{i}+d T_{1}\left(u_{2}\right) \notin R\left(T_{2}\right),
$$

implying that $\operatorname{dim}\left(R\left(T_{1}\right) / R\left(T_{2}\right)\right)=k+1$, which is false.

Lemma 5. For $p \in[1, \infty]$,

$$
\begin{aligned}
& \operatorname{dim} N\left(T_{p}\right)=\operatorname{dim}\left(\frac{R\left(T_{p}\right)}{R\left(T_{0, p}\right)}\right)=1, \\
& \operatorname{dim}\left(\frac{\mathcal{D}_{p}}{\mathcal{D}_{0, p}}\right)=2 .
\end{aligned}
$$

Proof. Since $M$ is disconjugate on $I_{a}$ and since $q>0$, it follows by Corollary 6.4 and Theorem 6.4 of Hartman [6] that there is a fundamental set of positive linearly independent solutions $y_{1}$ and $y_{2}$ of $M[y]=0$, called respectively the principal and nonprincipal solutions, such that $y_{1}^{\prime} \leq 0$ and $y_{2}^{\prime}>0$ on $I_{a}$. Additionally, $\lim _{t \rightarrow \infty} y_{1} / y_{2}=0$. Suppose $y_{p} \in N\left(T_{p}\right)$ for $p \in[1, \infty)$. We claim that $y_{p}$ must be a multiple of $y_{1}$. For if $y_{p}=c_{1} y_{1}+c_{2} y_{2}$ with $c_{2} \neq 0$, then

$$
\lim _{t \rightarrow \infty}\left|y_{p}(t)\right|=\lim _{t \rightarrow \infty}\left|y_{2}\left(c_{1} y_{1} / y_{2}+c_{2}\right)\right|=\left|c_{2}\right| y_{2} .
$$

Since $\left|y_{p}(t)\right|$ becomes arbitrarily close to a nondecreasing positive function, it cannot be in $L^{p}\left(I_{a}\right)$. This shows that $\operatorname{dim} N\left(T_{p}\right) \leq 1$, for $p \in[1, \infty]$, and if nontrivial, $N\left(T_{p}\right)$ is spanned by $\left\{y_{1}\right\}$. 
We next show that $N\left(T_{p}\right) \neq\{0\}$. If $r \in(1, \infty]$, then $R\left(T_{0, r}\right)$ is closed by Lemma 11 and from Theorem B (iv) and the fact that $L^{r}\left(I_{a}\right)=L^{r,}\left(I_{a}\right)^{*}$,

$$
\begin{aligned}
R\left(T_{0, r}\right) & ={ }^{\perp} N\left(T_{r^{\prime}}\right)=N\left(T_{r^{\prime}}\right)^{\perp}, \\
{ }^{\perp} R\left(T_{0, r}\right. & =N\left(T_{r^{\prime}}\right) .
\end{aligned}
$$

Also, by Lemma 1 (again), $R\left(T_{r}\right)=L^{r}\left(I_{a}\right)$. We now identify $M$ with $N\left(T_{r^{\prime}}\right), X$ with $L^{r^{\prime}}\left(I_{a}\right)$, and $X^{*}$ with $L^{r}\left(I_{a}\right)$. (2.2) and Lemma 2 then give that

$$
\operatorname{dim}\left(\frac{R\left(T_{r}\right)}{R\left(T_{0, r}\right)}\right)=\operatorname{dim} N\left(T_{r^{\prime}}\right) \leq 1 .
$$

Hence if $\operatorname{dim}\left(R\left(T_{r}\right) / R\left(T_{0, r}\right)\right)=0$, then $N\left(T_{r^{\prime}}\right)=\{0\}$, and by (2.3) and Lemma 4 $\operatorname{dim}\left(\mathcal{D}_{r} / \mathcal{D}_{0, r}\right)=\operatorname{dim} N\left(T_{r}\right)$. However, since we can find $C_{0}^{\infty}$ linearly independent functions $\phi_{1}, \phi_{2}$ with support in $I_{a}$ such that $\phi_{1}(a)=1=\phi_{2}^{\prime}(a)$ and $\phi_{1}^{\prime}(a)=0=$ $\phi_{2}(a)$ it must be the case that $\operatorname{dim}\left(\mathcal{D}_{r} / \mathcal{D}_{0, r}\right) \geq 2$. So $\operatorname{dim} N\left(T_{r}\right)=2$, which as we have seen from (2.1) is not possible given the properties of $y_{1}$ and $y_{2}$. This contradiction shows that $\operatorname{dim} N\left(T_{r^{\prime}}\right)=1$ for $r^{\prime} \in[1, \infty)$. If $p=r \in(1, \infty]$ by (2.4), then $\operatorname{dim}\left(R\left(T_{p}\right) / R\left(T_{0, p}\right)\right)=1$ and if we choose $r^{\prime}=p$, then $\operatorname{dim} N\left(T_{p}\right)=1$ for $p \in[1, \infty)$. If $p=\infty$, then $y_{1} \in N\left(T_{\infty}\right)$ since $y_{1}>0$ and $y^{\prime} \leq 0$, and so $\operatorname{dim} N\left(T_{\infty}\right)=1$. Since we have now established that $\operatorname{dim} N\left(T_{p}\right)=\operatorname{dim} N\left(T_{p}^{\prime}\right)$ for $p \in(1, \infty]$ by Lemma 4 and (2.3) $) \operatorname{dim}\left(D_{p} / D_{0, p}\right)=2$. By Lemma 3 . $\operatorname{dim}\left(\mathcal{D}_{1} / \mathcal{D}_{0,1}\right)=2$. Since $T_{1}$ is one-to-one on $\mathcal{D}_{1} \ominus N\left(T_{1}\right)$ we conclude that $\operatorname{dim}\left(R\left(T_{1}\right) / R\left(T_{0,1}\right)\right)=1$. The lemma is now established in all cases.

Proof of Theorem 1. (i) and (ii) is the assertion of Lemma 5. It follows that $\mathcal{D}_{p}=$ $\mathcal{D}_{0, p} \oplus \operatorname{span}\left\{\phi_{1}, \phi_{2}\right\}$. Since $\phi_{1}, \phi_{2}$ vanish at $\infty, \lim _{t \rightarrow \infty}\{y, z\}(t)=0$, which proves (iii).

Proof of Theorem 2. This is also obvious from (2.1) and the proof of Lemma 5 .

\section{REFERENCES}

[1] R. J. Amos and W. N. Everitt, On integral inequalities and compact embeddings associated with ordinary differential expressions, Arch. Rational Mech. Anal. 71-72 (1979/80), 15-39. MR 80f:47039

[2] R. C. Brown, The operator theory of generalized boundary value problems, Canad. J. Math. 29 (1976), 486-512. MR 54:1020

[3] N. Chernyavskaya and L. Shuster, A criterion for correct solvability of the Sturm-Liouville equation in the space $L_{p}(R)$, Proc. Amer. Math. Soc. 130(4) (2001), 1043-1054. MR 2002j:34040

[4] W. N. Everitt, A note on the Dirichlet condition for second-order differential expressions, Canad. J. Math., 28(2) (1976), 312-320. MR 55:3396

[5] S. Goldberg, Unbounded Linear Operators: Theory and Applications, McGraw-Hill Series in Higher Mathematics (E. H. Spanier, ed.), McGraw-Hill Book Company, New York, St. Louis, San Francisco, Toronto, London, Sydney, 1966. MR 34:580

[6] P. Hartman, Ordinary Differential Equations, Second Edition, Birkhäuser, Boston, Basel, Stuttgart, 1982. MR 83e:34002

[7] T. Kato, Perturbation theory for linear operators, Second Edition, Grundlehren der Mathematischen Wissenschaften, Band 132, Springer-Verlag, Berlin, Heidelberg, and New York, 1980. MR 53:11389

[8] M. A. Naimark, Linear Differential Operators, part II: Linear differential operators in Hilbert space, Frederick Ungar, New York, 1968. MR 41:7485

[9] T. T. Read, Exponential solutions of $y^{\prime \prime}+(r-q) y=0$ and the least eigenvalues of Hill's equation, Proc. Amer. Math. Soc. 50 (1975), 273-280. MR 51:13357 
[10] G. Rota, Extension theory of differential operators I, Comm. Pure and Applied Math. 11 (1958), 23-65. MR 20:3334

[11] W. Rudin, Functional Analysis, McGraw-Hill Series in Higher Mathematics (E. H. Spanier, ed.), McGraw-Hill Book Company, New York, 1973. MR 51:1315

Department of Mathematics, University of Alabama, Tuscaloosa, Alabama 354870350

E-mail address: dbrown@gp.as.ua.edu 